\title{
Estimation of thermo-hydrodynamic parameters in energy production systems using non-stationary signal processing
}

\author{
F.-M. Birleanu* - C. Ioana* - A. Serbanescu** - G. Serban*** - E. Sofron*** \\ (*): Grenoble INP - GIPSA-lab, Images-Signal Department, FRANCE \\ (**): Military Technical Academy, Bucharest, ROMANIA \\ $(* * *)$ : University of Pitesti, ROMANIA
}

\begin{abstract}
In order to control water flow in power generation systems, obtaining precise information about the parameters of the flow is important. The construction of such systems, as well as the operating conditions, impose the use of non-intrusive techniques. The use of ultrasounds allows solving this problem in a simple and elegant manner. The technique consists in sending a signal to be affected by the flow without sensibly interacting with it, and then extracting the desired parameters of the flow from the received signal. The paper first presents how the acoustic wave is altered by the propagation in the liquid environment, and then it studies the means to analyze the received signal in order to obtain from it information about two important parameters of the flow - the temperature of the water and the flow rate. Polynomial phase modeling is used, for obtaining a good estimation of the propagation times.
\end{abstract}

Keywords: Non-intrusive measurement, ultrasounds, temperature and flow rate estimation, polynomial phase modeling.

\section{INTRODUCTION}

The generation of electrical energy involves water flows through pipes. The parameters of these flows may provide information about the state of the system or of the pipe. In addition to that, the well-functioning of the system implies mantaining these parameters in between certain limits. Therefore, these flows must be controlled, and in order to close the loop of the control system, a method to obtain information about the flow is needed. This method is constrained by the fact that it must work while the system is on, and without affecting its functioning. A non-intrusive method is, therefore, needed. Ultrasounds promise to offer an elegant and efficient solution. In brief, the method involves the following: mounting the transducers on the outer walls of the pipe, transmitting a signal to be affected by the flow, and analyzing the received signal in order to obtain the flow parameters.

In this paper we will deal with the estimation of two very important parameters of the water inside the pipes - its temperature and its flow rate. We first show how the ultrasonic wave is modified by the interaction with the environment, in the contexts of determining each of these two parameters. We then present how the received signal is analyzed in the purpose of obtaining the information that allows estimating the desired thermo-hydrodynamic parameters.

The rest of the paper is organized as follows. Section 2 briefly presents the ultrasonic waves and the way they are affected by the temperature of the water through which they propagate, as well as by its flow rate. Section 3 presents the methods that were used to extract the desired information from the received signals. In section 4 we present the results we obtained using the described materials and methods. We conclude the paper with section 5 , in which we discuss the results.

\section{ULTRASOUND PROPAGATION THROUGH WATER}

The ultrasonic wave [1] is a pressure wave that propagates through an elastic medium with a frequency that is above 20 $\mathrm{KHz}$. That means that is consists in the propagation of a mechanical energy in a medium. The propagation medium of the ultrasonic wave is subject to a succession of over-pressures an under-pressures, and its constitutive particles are then animated by a back and forth movement on the propagating direction of the wave, as illustrated in Figure 1.

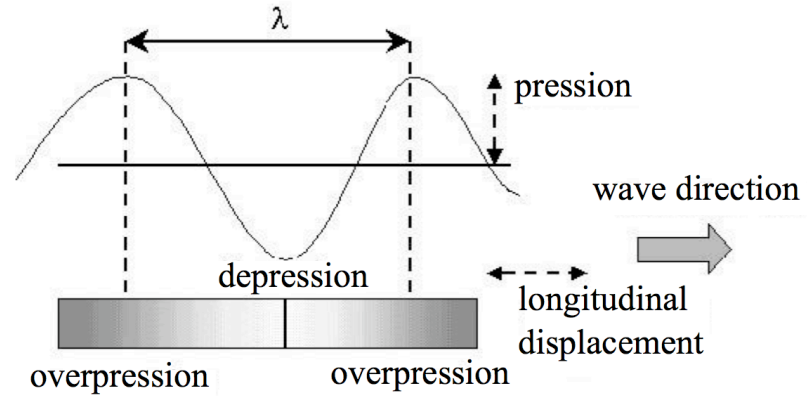

Figure 1. Illustration of the propagation of an acoustic wave

As any wave, the ultrasonic wave will interact with the propagation medium, being subject to attenuations, reflexions, refractions and diffractions.

We must note that before propagating through water the wave must first pass through the steel walls of the pipe. Ultrasound wave propagation through such solid, non-elastic 
media is possible, but is performed as transversal waves, with a much higher speed.

\section{II.1 Measuring temperature}

Sound propagation speed in pure water depends on some properties of the water, such as its local density [2], [3]. This speed can be found starting from the state equation of pure water. However, there are many simplified versions. The evolution law of the sound speed can be reduced to a dependency on the temperature of the fluid and, less importantly, to its pressure. The following equation presents the evolution of the sound speed in pure water with respect to the temperature, and it is obtained by polynomial interpolation of the experimental results:

$$
\begin{aligned}
& c=1.4024 \cdot 10^{3}+5.0384 \cdot T-5.8114 \cdot 10^{-2} \cdot T^{2}+ \\
& 3.3456 \cdot 10^{-4} \cdot T^{3}-1.4815 \cdot 10^{-6} T^{4}+3.1608 \cdot 10^{-9} \cdot T^{5}
\end{aligned} .
$$

Graphically, this evolution looks like in Figure 2.

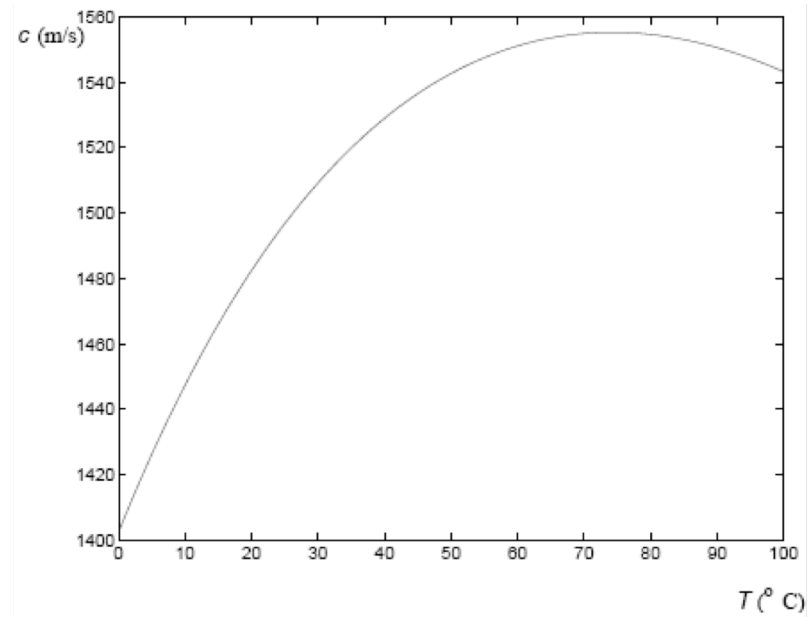

Figure 2. The dependence of the speed of sound with temperature

This strong dependence of the speed of sound with temperature will be used to determine the latter. There is, however, a problem. As seen in Figure 2, for a certain range of values for $c$, there are two possible values for $T$. Therefore, is seems impossible to determine $T$ knowing $c$. One possible solution to this problem would be estimating the level of noise in the received signal, as it is related to the temperature - a high level of noise would indicate a greater temperature.

\section{2 Measuring flow rate}

If an ultrasonic impulse is sent to a particle that is at rest, the echo produced by this impulse will be of the same shape. If the particle gets off the emitter, the ultrasonic wave will need a longer time to cover the way to the particle and back. This variation of the length of the route, caused by the variation of the distance to be covered, is called propagation delay. The echos of a particle are always the same when the particle is at rest, that is, when there is no propagation delay. The echos have the same relative phase, which means that there is no phase difference. In case of superposition of a second echo on a particle that gets off the receiver, this echo will be delayed with respect to the first one.

A propagation delay corresponds to a modification of distance, so if we measure the delay and knowing the speed of sound, we can evaluate how far the particle moved, and as we know the delay between the pulses, we can compute the speed of the particle [4]. This measurement principle is illustrated in Figure 3.

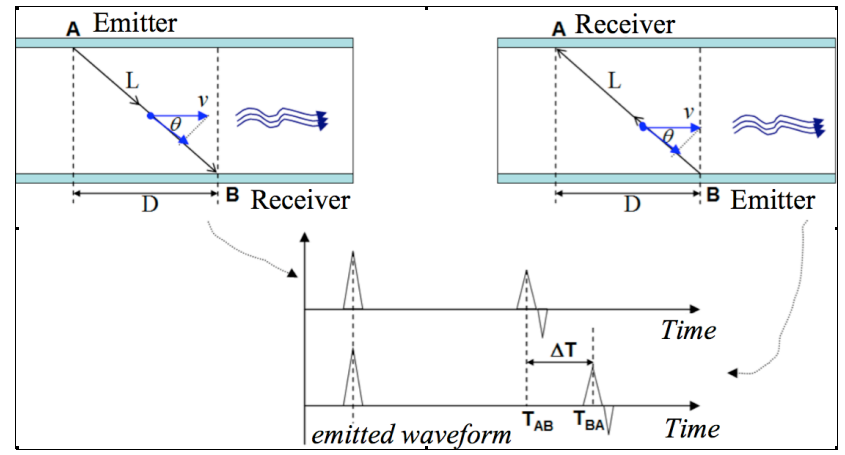

Figure 3. The flow rate measurement principle

The flow rate measurement principle using the difference of propagation times consists in performing transmissionsreceptions in the flow direction, as well as in the reverse direction. In the first case, the speed of fluid particles will affect the covered time, $T_{A B}$, as follows:

$$
T_{A B}=\frac{L}{c+v \cos \theta}+2 T_{\text {paroi }}+2 T_{\text {capteur }},
$$

where $c$ is the speed of sound, $v$ is the average speed of the fluid, $\theta$ is the angle between the propagating direction and the horizontal, $T_{\text {paroi }}$ is the propagation time through the walls and $T_{\text {capteur }}$ is the transit time inside the transducers. We may note that the speed of the fluid acts as an additional apparent speed that is added when the emission is performed in the direction of the flow. In the other case, when the emission is performed in the reverse direction, this apparent speed diminishes the global speed of sound. All these lead to a propagation time like:

$$
T_{B A}=\frac{L}{c-v \cos \theta}+2 T_{\text {paroi }}+2 T_{\text {capteur }}
$$

Using these two measures of the arrival time, the speed of the fluid can be expressed as:

$$
v=\frac{L^{2}}{2 D}\left(\frac{\Delta T}{t_{A B} \cdot t_{B A}}\right) \text {, with } \Delta T=T_{B A}-T_{A B},
$$

where $t_{A B}=T_{A B}-2 T_{\text {paroi }}-2 T_{\text {capteur }}$ and $t_{B A}=T_{B A}-2 T_{\text {paroi }}-2 T_{\text {capteur }}$. The volumic flow rate is then computed starting from the average speed, using:

$$
Q=\frac{\pi D_{\mathrm{int}}^{2}}{4} \cdot \frac{L^{2}}{2 D} \cdot\left(\frac{\Delta T}{t_{A B} \cdot t_{B A}}\right) \cdot \frac{1}{K_{H}},
$$


where $D_{\text {int }}$ is the inner diameter of the cylinder and $K_{H}$ is the hydraulic coefficient, computed starting from the Reynolds number [5], Re, using:

$$
K_{H}=\frac{2 n+1}{n}, \text { with } \frac{1}{n}=0.25-0.023 \cdot \log (\mathrm{Re}) .
$$

What has been discussed so far in this section shows that estimating the propagation times gives us access to the flow rate of the fluid, as well as to its temperature. We will show in the next section a signal analysis method that is focused on estimating as accurate as possible these arrival times.

\section{II.3 Experimental setup}

The block diagram of the experimental setup used for measuring the temperature is presented in Figure 4.

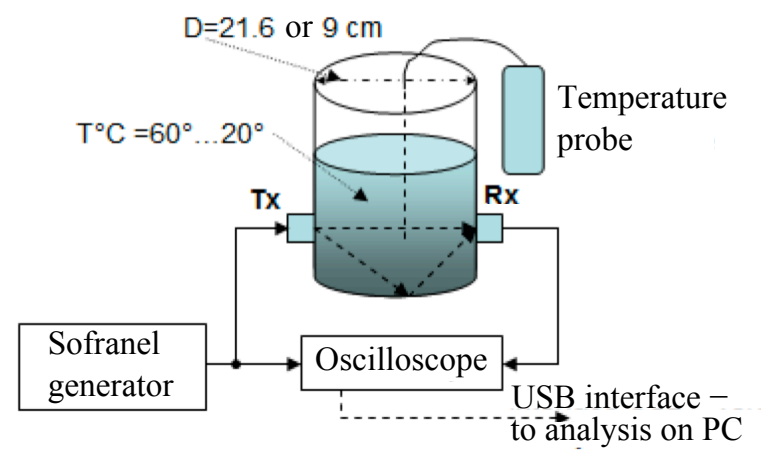

Figure 4. Experimental setup for measuring the temperature

We used hot tap water, and we let it get cold progresively. The non-intrusive temperature measurement procedure was repeated every ten minutes. The temperature was also measured using a thermometer that was introduced inside the water, at the level of the transducers.

The flow rate was measured using an experimental setup whose block diagram in presented in Figure 5.

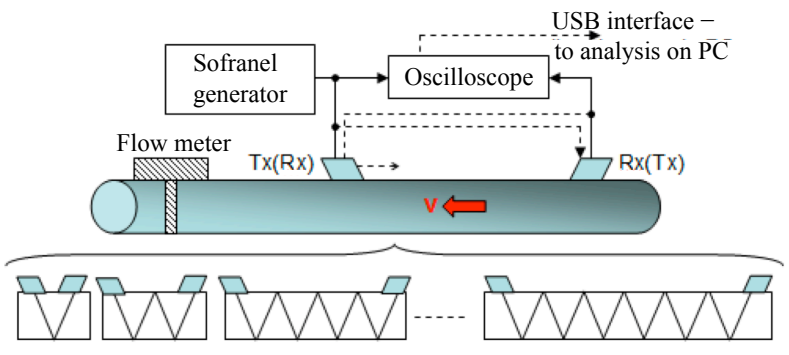

Figure 5. Experimental setup for measuring the flow rate

Several positions for the transducers have been tried, in order to maximize the received energy, using the available equipment ( - the sampling frequency of the oscilloscope was $10 \mathrm{MHz})$.

\section{THE ANALYSIS OF THE RECEIVED SIGNALS}

A brute visual analysis of the received signal shows that before the expected signal we obtain a signal that corresponds to the propagation (with a speed that is much greater than the speed in water) through the steel walls of the pipe. The fact that this separation and detection of the signals can be done visually suggests that we could analyze them using methods that are based on the analysis of the temporal shape of the received pulses. We must note, however, that noise and distorsions due to the propagation pose problems to this class of methods.

A possible solution consists in considering the envelope of the received pulses as a suite of phase modulations and trying to estimate them by a polynomial phase modeling. This concept, introduced in [6], has the advantage of providing a representation space that is more robust to perturbations of any kind: noise, propagation, multiple routes, etc. More precisely, the envelope of the received pulses is characterized by the polynomial coefficients whose values stay stable in the presence of perturbations.

Mathematically, a warping operator is defined as a unitary transform $\mathrm{U}$ on $l^{2}(\hat{\mathrm{A}})$, whose effect is $x$-axis deforming :

$$
(\mathbf{U} s)(x)=\mid w^{\prime}(x)^{1 / 2} s[w(x)],
$$

where $w$ is a smooth, one-to-one function, comprising a large subclass of unitary transformations. Generally, these functions, called warping functions, are chosen to ensure the "linearization" of signal time-frequency behavior. To reduce the error propagation effect, the warping operator is defined as as :

$$
\mathbf{w}_{\mathbf{K}}: t \stackrel{\mathbf{w}_{\mathbf{K}}}{\longrightarrow} t_{w}^{(K)}=\mathbf{w}_{\mathbf{K}}(t)=\left(\frac{t}{\left|\hat{a}_{K}\right|}\right)^{1 / K},
$$

where $\hat{a}_{k}$ is the $k^{\text {th }}$ order estimated polynomial coefficient.

Polynomial modeling is done using higher order ambiguity functions [6]. In our context, this modeling starts with evaluating the ambiguity function of order 6 that allows, through an estimation of maxima, the estimation of the polynomial coefficient of order 6 . Its influence on the analyzed signal is removed using a warping procedure, thus avoiding the propagation of the estimation errors that might appear, to lower orders. This procedure, that is called WarpCom, is illustrated in Figure 6 and is followed until the estimation of the first order coefficient is done.

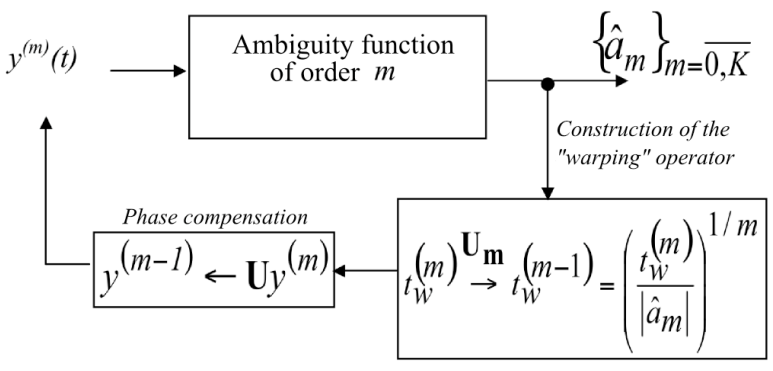

Figure 6. Block diagram of the WarpCom procedure

For the pulse train in Figure 7, the polynomial modeling of the emitted and, respectively, of the received signal are 
illustrated in the same figure. We may note the quasiinvariance we obtained thanks to the polynomial coefficients.
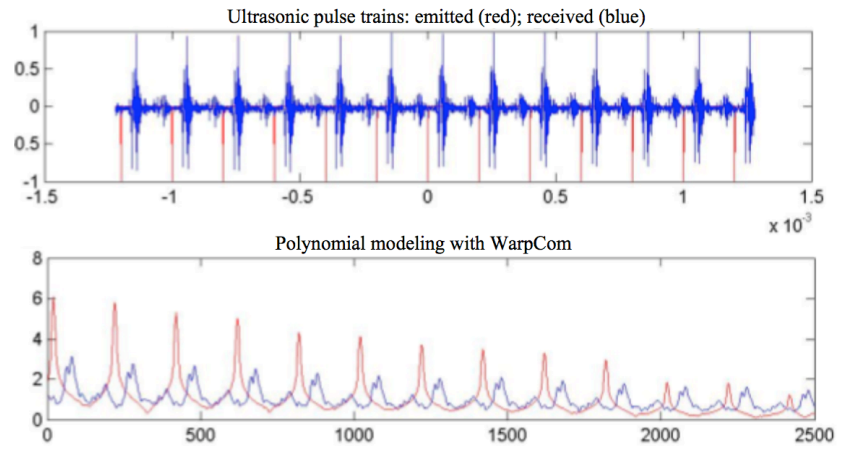

Figure 7. Polynomial modeling for a pulse train

In order to reduce the effect of modeling errors, we may proceed to an averaging on more pulses of the results of the polynomial modeling.

\section{RESUlts}

The method we presented offers good results for estimating the speed of sound in terms of temperature. The results obtained by estimation are illustrated comparatively together with the thoretical ones in Figure 8.

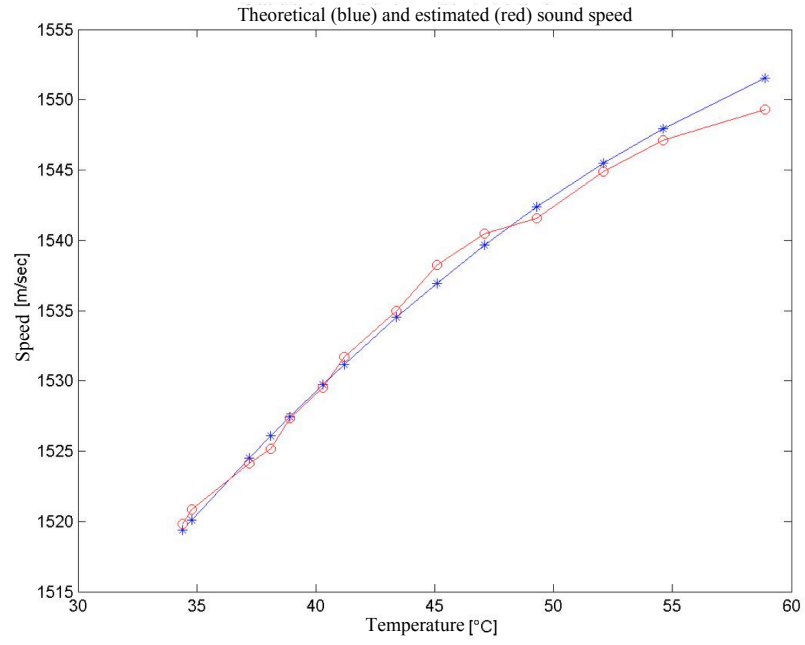

Figure 8. Results obtained for the temperature

As the desired parameter is in fact the temperature, it can be found starting from the speed of sound and using the curve in Figure 2. As mentioned before, for a certain range of speeds there is no bi-univocal correspondence between speed and temperature - thus an estimation of the level of noise (that increses with temperature) might discriminate between the two cases.

Figure 9 presents the flow rate estimations using the WarpCom method and the experimental setup and procedure that have been presented previously.

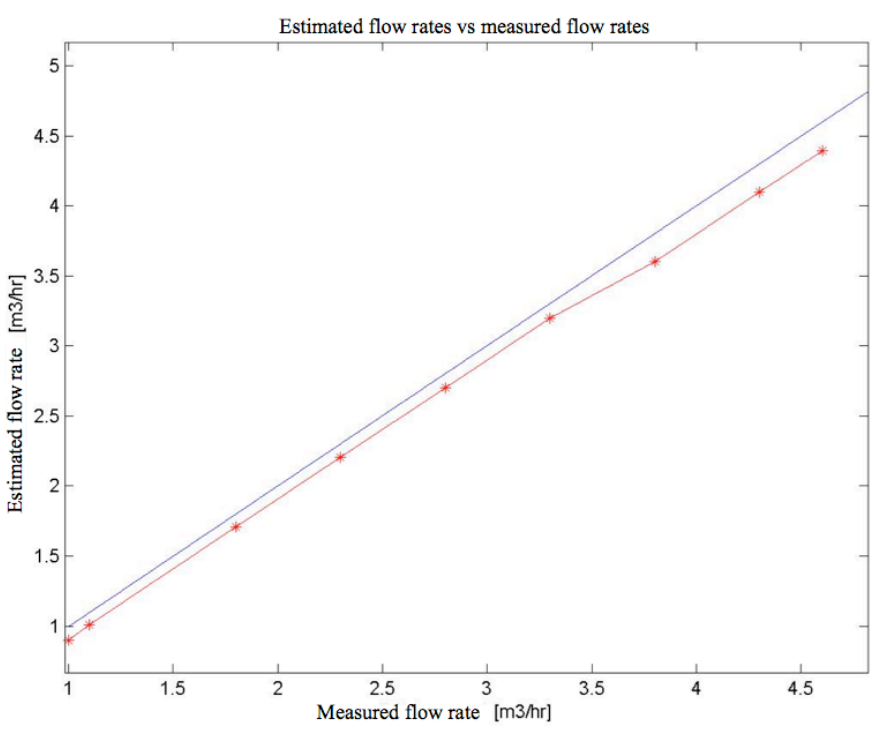

Figure 9. Results obtained for the flow rate

If we compare the results obtained by estimation with the reference ones, we notice that the estimation performance stays almost unchanged for a flow rate between 1 and $3.4 \mathrm{~m}^{3} / \mathrm{h}$. Above this value, there are more and more errors, having as main cause the vibrations of the pipe, that are present for a flow rate that is greater than $3 \mathrm{~m}^{3} / \mathrm{h}$. We believe that these errors are not due to the methodology, but rather to the experimental physical setup, that allows that these vibrations cause vibrations on the transducers, leading to the observed errors in estimating the propagation times.

\section{CONCLUSION}

The results obtained by estimating the propagation times using the WarpCom method, correlated with the physics of the experimental setup, allow good estimations for the hydraulic parameters, as the flow rate and the temperature of the water. The results we obtained for real-world signals show the feasibility of the principle of estimating the hydraulic parameters by measuring the differences of propagation times.

\section{REFERENCES}

[1] E.P. Papadakis, "Ultrasonic instruments and devices," Academic Press, 1999.

[2] D.R. Raichel, "The Science and Applications of Acoustics. Second Edition," Springer Science+Business Media, Inc., 2006.

[3] F. Everest, "The Master Handbook of Acoustics," McGraw-Hill, New York, 2001.

[4] L. C. Lynnworth, "Ultrasonic Measurements for Process Control: Theory, Techniques, Applications," Academic Press, 1989.

[5] M. Lesieur, "Turbulence in Fluids," Springer, 2008.

[6] C. Ioana, A. Quinquis, "Time-Frequency Analysis using Warped-Based High-Order Phase Modeling," EURASIP Journal of Applied Signal Processing, vol. 2005, no. 17, pp. 2856-2873, September (2005). 